\title{
Effects of Advance Care Planning on End-of-Life Decision Making: A Systematic Review and Meta-Analysis
}

\author{
Minju Kim, R.N., Ph.D. and Jieun Lee, R.N., Doctoral Student \\ College of Nursing, Dong-A University, Busan, Korea
}

Purpose: The purpose of this systematic review and meta-analysis was to investigate the effects of advance care planning on end-of-life decision-making. Methods: Databases including RISS, KISS, KMbase, KoreaMed, PubMed (MEDLINE), Embase, and CINAHL were searched for studies that examined the effects of advance care planning interventions. The inclusion criteria were original studies in English or Korean; adults $\geq 18$ years of age (population); advance care planning (intervention); completion of advance directives (AD) or advance care planning (ACP) (outcomes); and randomized or non-randomized controlled trials (RCTs and non-RCTs, respectively) (design). Study quality was measured using the checklists of the Joanna Briggs Institute. Meta-analyses were conducted with the Comprehensive Meta-Analysis program. Results: Nine RCTs and nine non-RCTs were selected for the final analysis. The effect sizes (ES) of the outcome variables in nine RCTs were meta-analyzed, and found to range from 0.142 to 0.496 for the completion of $\mathrm{AD}$ and $\mathrm{ACP}$ ( $\mathrm{ES}=0.496,95 \% \mathrm{CI}: 0.157 \sim 0.836)$, discussion of end-of-life care ( $\mathrm{ES}=0.429$, 95\% CI: $-0.027 \sim 0.885$ ), quality of communication ( $E S=0.413$, 95\% CI: $0.008 \sim 0.818$ ), decisional conflict ( $\mathrm{ES}=0.349,95 \% \mathrm{CI}:-0.059 \sim 0.758)$, and congruence between preferences for care and delivered care ( $\mathrm{ES}=0.142,95 \%$ CI: $-0.267 \sim 0.552)$. Conclusion: ACP interventions had a positive effect on the completion of $\mathrm{AD}$ and $\mathrm{ACP}$. To apply $\mathrm{AD}$ or $\mathrm{ACP}$ in Korea, it is necessary to develop ACP interventions that reflect aspects of Korean culture.

Key Words: Palliative care, Decision making, Advance care planning, Systematic review, Meta-analysis
Received January 13, 2020

Revised April 4, 2020

Accepted April 28, 2020

\section{INTRODUCTION}

\section{Background}

The end-of-life decision making process provides an opportunity to understand the types of care that patients and their families wish to receive or not receive in the face of a lifethreatening illness. However, discussing death and planning for end-of-life care with terminally ill patients-let alone with healthy family members-is challenging in Korean culture, where people are reluctant to discuss the grave and serious topic of death [1]. Nonetheless, sharing thoughts and plans regarding death and end-of-life care allows patients to avoid unnecessary life-sustaining care [2] and to receive the types of care that they would prefer.

In Korea, most end-of-life care decisions are made by family members after a patient's condition has already become critical [3-5], and these decisions are accepted without considering the wishes of the patient [6]. These decision-making patterns are rooted in a culture where family-based decision- 
making is considered normal [4,7]. A study at a general hospital showed that $46.2 \%$ of cases in which cardiopulmonary resuscitation (CPR) was discontinued involved patients whose consciousness was compromised [8]. This means that roughly half of the patients were unable to make critical decisions for themselves, such as discontinuing CPR, at the time when those decisions were made. In other words, when the time comes to make end-of-life care decisions, the responsibility is often in the hands of others, such as family members and medical staff, regardless of the patient's wishes. Therefore, it is important for patients to set up advance care planning when they are still able to make their own decisions.

In 2018, the Act on Hospice and Palliative Care and Decisions on Life-Sustaining Treatment for Patients at the End of Life (hereinafter referred to as the Act on Decisions on Life-Sustaining Treatment) came into force, with the goal of protecting human dignity and values by respecting the selfdetermination of terminal patients [9]. This act authorized patients to create advance directives and life-sustaining treatment plans. The National Agency for Management of LifeSustaining Treatment released information on the status of the life-sustaining treatment system on September 2, 2019, according to which there had been 62,546 reported decisions to withdraw life-sustaining treatment [10]. Only $32 \%$ of these patients had prepared advance directives $(20,139)$ or lifesustaining treatment plans (725 cases) and most cases were decided based on statements made by the family of the patient (20,129 cases) or through agreement with the patient's family (21,553 cases) [10]. Even with the increased social recognition of the importance of end-of-life care and life-sustaining treatment planning since the enactment of the Act on Decisions on Life-Sustaining Treatment, decisions were still being made by the family more often than by the patients themselves [6]. Concerns have been raised that even as various kinds of end-of-life care decisions, such as withdrawing life-sustaining treatment, are being made, these decisions may reflect the will of family members, not that of the patient [11,12], and that self-determination rights of patients are not being protected with regard to end-of-life care decisions in clinical settings $[8,13,14]$. Intervention efforts to help patients plan for endof-life treatment options will be necessary to address these issues.
Other countries have implemented many different programs to help patient prepare advance care planning, such as the "Let me decide," "Let me talk," and "Respect choice" programs and the physician order for life-sustaining treatment (POLST) approach. The 'Let me decide' program provides patients and their families with education on writing advance directives and proved to be effective in reducing the number of hospitalizations and medical costs [15].

The "Let me talk" program involves exchanging opinions on life, illness, and preferred end-of-life treatments and has demonstrated positive results in promoting advance directives and improving quality of life [16]. The "Respecting choices" program, which was designed to promote creating advance care planning $[17,18]$, helps patients better understand advance care planning through interviews and takes into account their goals of end-of-life care. The program also encourages patients to communicate with their loved ones about their desires and to put the discussions in writing. A meta-analysis of the effects of the "Respecting choices" program reported that the program contributed to better alignment of the opinions of patients and their representatives [17]. The POLST approach is a communication program emphasizing shared decision-making [19] that was developed to help physicians communicate more effectively with patients regarding their treatment preferences for end-of-life care. The POLST form was designed to function as a guideline for treatment if the patient's status changes, such as in circumstances when CPR, treatment interventions, antibiotics, or nutritional interventions are needed. Hickman et al. [19] showed that the POLST program was effective in reducing the use of life-sustaining treatment and increasing the use of hospice care. As outlined above, there are many ways to help patients write advance directives, and the effects of interventions varied across studies. All of the programs used in these studies focused on communication with the aim of enhancing patients' ability to make decisions.

We found very limited Korean research investigating the application of interventions in advance care planning. Systematic literature reviews will be crucial for the development of effective intervention methods to promote the preparation of advance care planning that are applicable in Korea. It will also be necessary to study ways to respect patients' decisions and to communicate effectively with them regarding the completion 
of advance directives.

\section{Purpose}

The purpose of this study was to analyze the effects of intervention programs developed to help decision-making related to end-of-life care in Korea and abroad through the preparation of advance care planning. In this study, we analyze existing intervention programs that aim to promote the completion of advance directives, and thereby will provide a foundation for establishing effective intervention programs for Korean patients at the end of life.

\section{METHODS}

\section{Study design}

This study is a systemic literature review and meta-analysis of the effects of programs aiming to assist decision-making regarding end-of-life care on advance care planning.

\section{Literature selection criteria}

\section{1) Inclusion criteria}

A literature search was conducted based on the population, intervention, comparison, outcomes, study design (PICOS) approach. All patients receiving end-of-life care, except for children, were selected as participants. All advance care planning intervention programs developed to assist in decision-making were included as subjects in the search. Non-intervention groups of patients who did not participate in these programs or received usual care were selected as comparison groups. The primary outcome variables of the interventions were completion of advance directives and advance care planning, discussion of end-of-life care, and quality of communication. Secondary outcome variables were decisional conflict, confidence of the patient's legal representative in decision-making, congruence between the patient and the patient's legal representative regarding the preferred treatment, and congruence between preferences for care and delivered care. The study design was limited to experimental studies only. The languages were limited to English and Korean, and only studies published before December 27, 2018 were included.

\section{2) Exclusion criteria}

Studies not published in Korean or English, studies only published as an abstract or a poster, academic conference materials, books, and studies where the full text was unavailable were excluded. Additionally, studies in which the outcome variables were preferences and knowledge regarding end-oflife care were excluded, as well as those in which the effect size could not be estimated.

\section{Literature search}

The databases used to search the international literature were PubMed (MEDLINE), Embase, and CINAHL, while RISS, KISS, KMbase, and KoreaMed were used to search the Korean literature. Studies were searched using the PICOS approach. The two researchers discussed and agreed on the search strategy and confirmed their congruence through a preliminary search before performing the actual search. The researchers independently searched all of the databases. Manual searching of reference lists was performed to find any additional literature.

The Korean literature was searched using the following terms (translated): ('end-stage' OR 'end-of-life') AND ('advance treatment plan*' OR 'advance care*' OR 'advance medicine*' OR 'advance life-sustaining medicine*' OR 'advance will*' OR 'advance consensus*' OR 'advance application*' OR 'advance directives*' OR 'advance rejection of treatment' OR 'advance notice' OR 'advance request*' OR 'advance decision*' OR 'advance choice testament' OR 'death choice testament' OR 'life testament*' OR 'living testament*' OR 'living will' OR 'decision making' OR 'life-sustaining' OR 'life prolonging' OR 'lifespan extension' OR 'survival*' OR 'do-notresuscitate order' OR 'non-execution of CPR' OR 'palliative*' OR 'hospice' OR 'shared decision making' OR 'common decision making' OR 'communication' OR 'conversation'). The international literature was searched using the following terms: ('advance care direct*' OR 'advance care plan*' OR 'advance directive*' OR 'advance health care plan*' OR 'advance healthcare plan*' OR 'advance medical direct*' OR 'advance medical plan*' OR 'DNH' OR 'DNR' OR 'living will*' OR 'wish') AND ('end of life' OR 'hospice' OR 'life prolonging' OR 'life support' OR 'life sustaining' OR 'palliative' OR 'terminal care') AND ('decision making' OR 'shared 
decision making' OR 'communication' OR 'conversation' OR 'end of life discussion'). The search criteria were limited to human studies that were in English (as well as Korean in some databases).

\section{Quality evaluation of the literature}

We performed a critical appraisal of the quality of the literature using the quality evaluation tools developed by the Joanna Briggs Institute (JBI) [20]. The researchers independently reviewed the quality of articles and discussed and analyzed the results to come to a final conclusion. Different quality assessment tools were used based on the study design. Randomized controlled trials (RCTs) were evaluated using the Checklist for Randomized Controlled Trials. This tool contains 13 items, including randomization used for assignment of participants, concealment of allocation to treatment groups, similarity of participants, participant blinding, treatment provider blinding, assessor blinding, identical treatment other than the intervention of interest, adequate treatment of completion of followup and difference between groups, analysis of participants in the randomized groups, identical measurement of outcomes, reliability of measurements, appropriateness of statistical analysis, and appropriateness of trial design. The Checklist for Quasi-Experimental Studies (non-randomized experimental studies) was used to appraise non-randomized studies. This tool comprises nine items, including a clear distinction of cause and effect, similarity of participants, control of participants, existence of a control group, measurement of outcomes before and after the intervention/exposure, adequate treatment of completion of follow-up and difference between groups, identical measurement of outcomes, reliability of measurements, and appropriateness of statistical analysis. Each item was rated as "yes", "no", "unclear", or "not applicable".

\section{Data coding}

All the searched literature was entered into the reference management software Endnote X8. The studies included in the final analysis were arranged into a table by their characteristics, with reference to the characteristics analyzed in the existing literature. Author, year, country, setting, study design, participants, number of participants, intervention, outcome measurement timeframe, and outcome variables were recorded in the coding sheet. One of the researchers filled out the coding sheet, while the other checked for potential errors.

\section{Data analysis}

Based on the statistics (mean $\pm \mathrm{SD}, \mathrm{P}$ ) reported in the studies selected for the systematic review, effect sizes (d) were calculated using Microsoft Excel Effect Size Computation Program. The meta-analysis of the literature was conducted using the Comprehensive Meta-Analysis software version 3 (Biostat, Englewood, NJ, USA).

\section{1) Calculation of effect size}

Effect sizes were calculated using the standardized mean difference (SMD) to standardize the inconsistent results reported by the studies. An effect size of 0.2 or less represents a small effect, 0.5 indicates a medium-sized effect, and 0.8 and higher corresponds to a large effect [21].

\section{2) Analysis of heterogeneity}

The chi-square test (Q statistics) and Higgin's $\mathrm{I}^{2}$ were used to confirm the heterogeneity of the data. In Q statistics, the null hypothesis of a homogenous effect size is tested, where a fixed-effects model is applied for $\mathrm{P}>0.10$ and a randomeffects model is applied for $\mathrm{P}<0.10$ [22]. An $\mathrm{I}^{2}$ between $0 \%$ and $40 \%$ implies that heterogeneity might not be important, an $\mathrm{I}^{2}$ between $30 \%$ and $60 \%$ implies medium-level heterogeneity, and an $\mathrm{I}^{2}$ of $75 \%$ to $100 \%$ means significant heterogeneity [23]. A forest plot was constructed for the visual evaluation of heterogeneity.

\section{3) Selection of analysis model}

A fixed-effects model is appropriate when there is minimal heterogeneity in the effect size, while a random-effects model is suitable for high heterogeneity. A fixed-effects model was applied in this study, as low heterogeneity was found for all variables, with a $\mathrm{P}>0.10$.

\section{4) Publication bias}

Publication bias occurs when the outcome of a study influences the decision of whether to publish it [23]. The funnel plot and trim-and-fill methods were used to check for publication bias. The funnel plot method has long been used to evaluate 
the possibility of missing outcomes in meta-analyses [23], using a dispersion chart with the $\mathrm{x}$-axis displaying the effect size and the $y$-axis displaying the sample size [22]. Smaller studies are distributed widely at the bottom of the graph, while larger studies are distributed narrowly at the top [23]. If the graph forms a funnel-shaped distribution, the possibility of publication bias is relatively low. The trim-and-fill method is a way of estimating how many studies are in the non-symmetric area of a funnel plot, by trimming the studies causing funnel plot asymmetry, using the symmetric area to estimate the true center of the funnel, and then replacing the omitted studies and their missing counterparts at the center [24].

\section{RESULTS}

\section{Selection of literature}

The literature for the systematic review was selected in accordance with the Preferred Reporting Items for Systematic Reviews and Meta-Analysis (PRISMA) guidelines. During the literature search, the researchers evaluated the appropriateness of each other's search strategy through a preliminary search.
The researchers independently reviewed the literature at each stage of the literature selection process and selected the final list through discussion. The search results found a total of 1,293 Korean studies (362 from RISS, 102 from KISS, 124 from KMbase, and 705 from KoreaMed) and 3,334 international studies (2,574 from PubMed [MEDLINE], 243 from Embase, and 517 from CINAHL). The total number of domestic and overseas studies was 4,668 after adding 42 manually searched studies. Among them, 1,053 studies overlapped according to the reference management software, reducing the net number of studies to 3,615. Next, 132 studies were selected after reviewing titles and abstracts, ruling out 3,483 studies that did not meet the selection criteria. Upon reviewing the full text, 105 studies were additionally eliminated, including three studies that did not meet the participant criteria, 19 studies that did not discuss interventions for advance care planning, 46 studies with irrelevant outcome variables, 20 studies that were qualitative, protocols, or systematic literature reviews, three studies for which the full text could not be accessed (poster or nonpublished dissertations), and 14 studies that did not clearly report statistical values. The quality appraisal of the 27 studies that met the initial selection criteria resulted in the removal

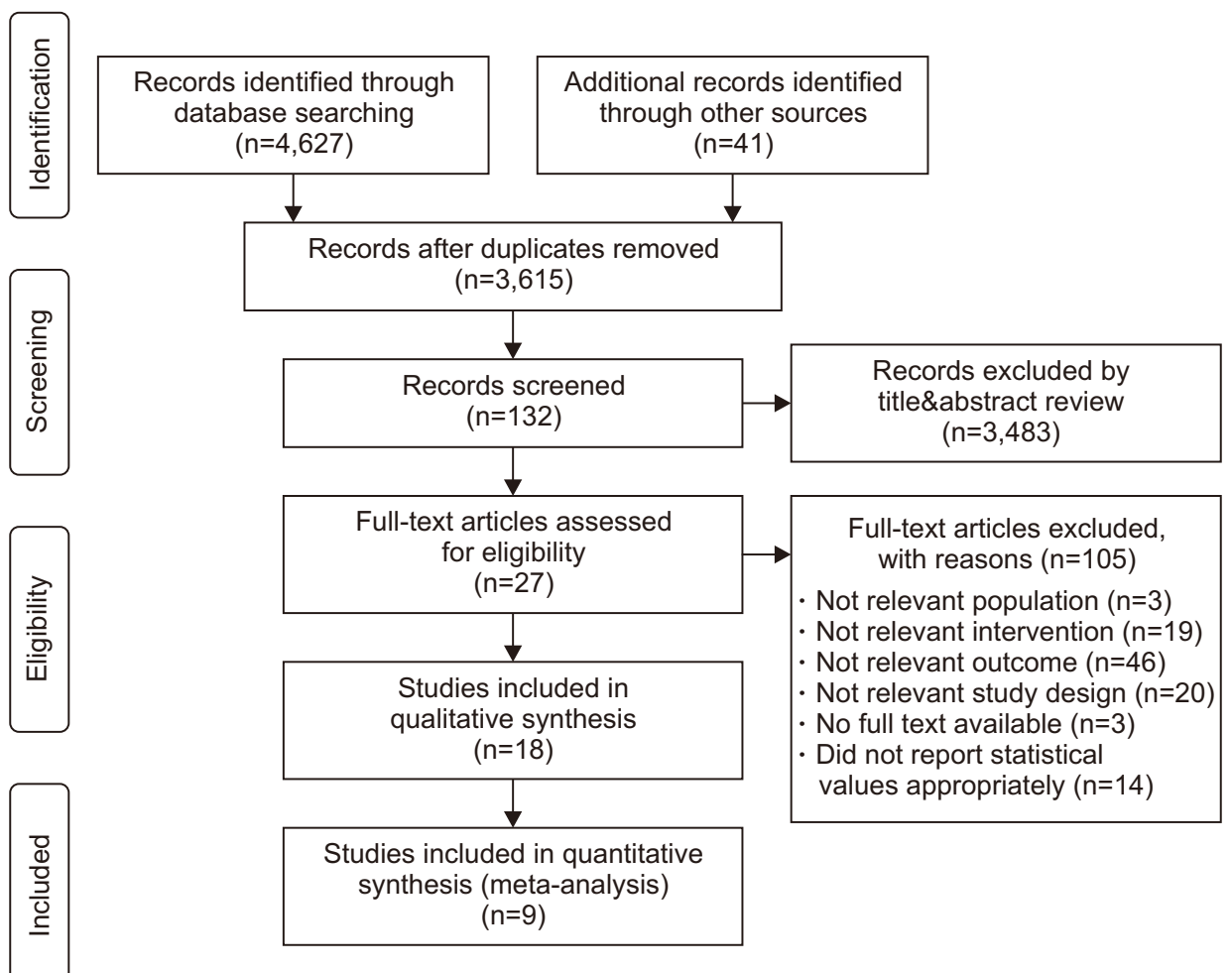

Vol. 23 - No. $2 \cdot 2020$
Figure 1. PRISMA flowchart. PRISMA: preferred reporting items for systematic reviews and meta-analysis. 


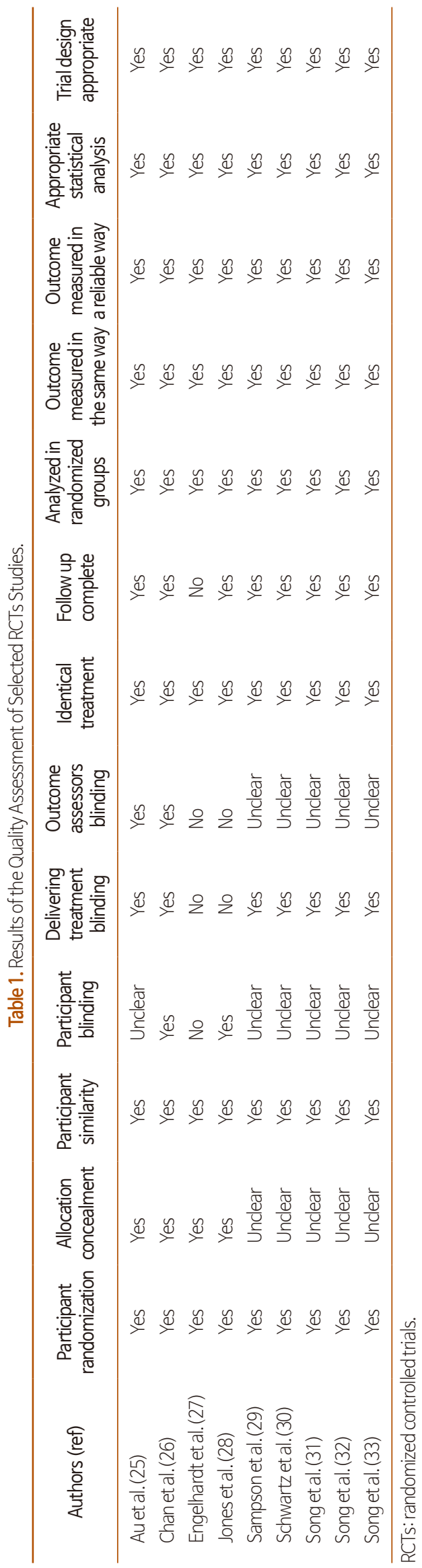

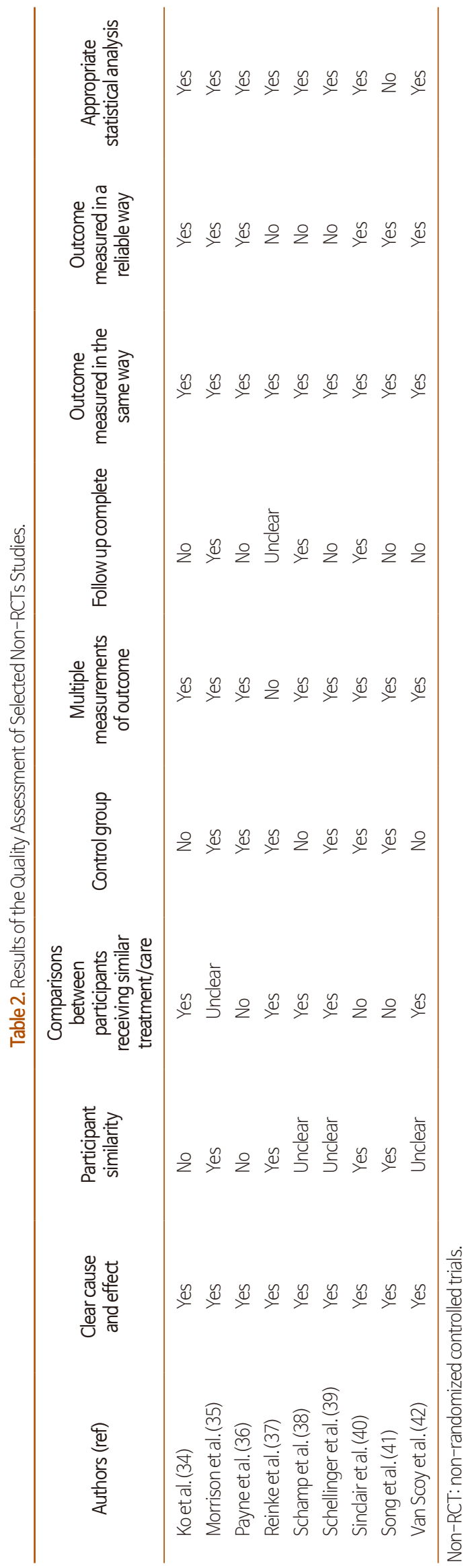

https://doi.org/10.14475/kjhpc.2020.23.2.71 
of nine other studies, leaving nine RCTs and nine non-RCT studies as the final subjects of the systematic review. Metaanalyses were only performed for the nine RCTs. All of the finally selected studies were conducted in countries other than Korea, because no domestic studies met the final inclusion criteria, even though some Korean studies appeared in the initial search (Figure 1).

\section{Quality appraisal outcomes}

The JBI quality appraisal tool does not suggest criteria for evaluating the overall quality of studies; instead, it only allows an item-by-item evaluation. Through discussions, the researchers established criteria for selecting the final studies; specifically, studies with a randomized control group were only included if at least nine out of 13 items (70\%) were assured of quality, and non-randomized experimental studies were only included when at least six out of nine items (roughly 70\%) were assured of quality. The quality of the 27 studies initially included in the systematic review was assessed. Two RCTs did not meet the criteria, leaving nine studies for the final analysis (Table 1). Of the 16 non-RCT studies, five were excluded, leaving nine non-RCTs for the final analysis (Table 2).

\section{RCTs}

An analysis of the characteristics of the included RCTs (Table 3) showed that the studies were published between 2002 and 2018, including three that were published before 2010. The patients' conditions included chronic obstructive pulmonary disease, congestive heart failure, cancer, dialysis, and severe dementia, and the participants included community-dwelling patients, elderly patients, and homeless persons. The number of participants in each study was between 32 and 376.

The intervention programs and the number of studies they appeared in were as follows: a patient-specific feedback form in one study [25]; an advance care planning program (a revised version of the "Let me talk" program) in one study [26]; an advanced illness coordinated care program in one study [27]; an advance care planning intervention in two studies $[28,29]$; the respecting choices intervention in one study [30]; the end-of-life planning intervention in one study [31]; and the Sharing Patients' Illness Representations to Increase Trust (SPIRIT) intervention in two studies [32,33]. Most of the in- tervention programs included discussions, conversations, and consultations as basic elements [26-29,32,33]. Conversations with the patient or the patient's legal representative were held to confirm the patient's health status and their preferences regarding end-of-life care. Some comprehensive interventions added education to the basic discussions and conversations [30,31]. The education provided information and resources for advance care planning. In the patient-specific feedback form intervention, physicians provided a feedback form [25] to patients and patient's legal representatives to let them know how well the physician understood the patient's desires for end-oflife care after having conversations with the patient. In most of the interventions, the outcomes were recorded in the form of advance directives or documentation demonstrating the wishes of the patient [27-31], or the patients were connected with physicians so that their wishes could be documented [26]. The interventions consisted of one to four sessions that lasted from 45 minutes to 1 hour.

The outcome variables of the studies were required to meet the selection criteria of this study, including the completion of advance directives and advance care plans, discussion of endof-life care, quality of communication, decisional conflict, the patient's legal representative's confidence in decision-making, and congruence regarding preferred care between the patient and the patient's legal representative. No RCT studies analyzed congruence between preferences for care and delivered care as an outcome variable.

\section{Non-RCT studies}

The nine non-RCT studies (Table 4) were published between 2005 and 2017, with two studies published before 2010 and seven studies published after 2010. The participants' illnesses included chronic obstructive pulmonary disease, heart failure, and chronic kidney disease, and the participants were lowincome older adults, long-term care residents, and community dwellers. The number of participants in each study was between 19 and 1,894.

The intervention programs and the number of studies they appeared in were: a stage-matched motivational interviewing intervention in one study [34], a social work intervention in one study [35], a protection motivation theory intervention in one study [36], a patient-specific feedback form in one study 


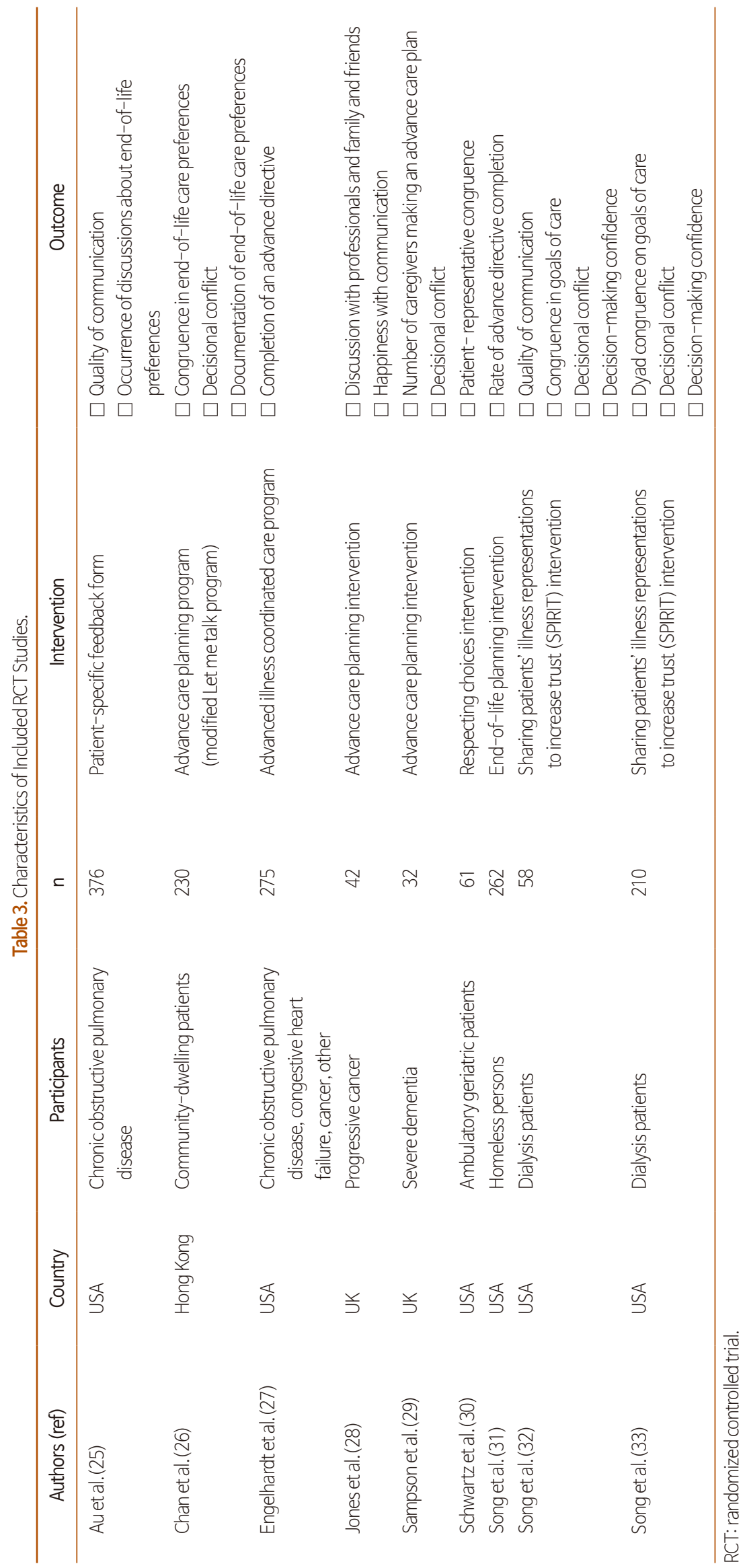




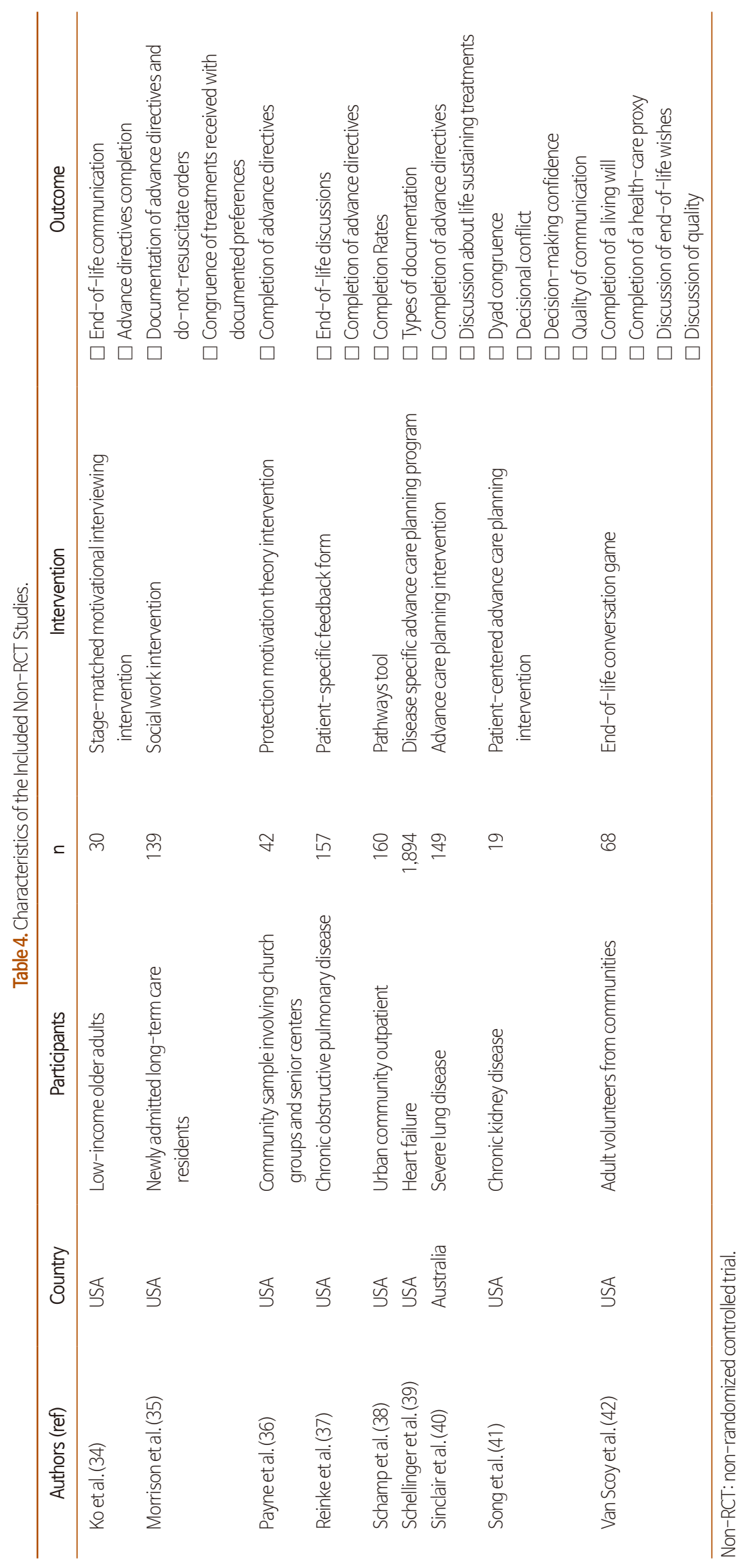


[37], a pathway tool in one study [38], a disease-specific advance care planning program in one study [39], an advance care planning intervention in one study [40], a patientcentered advance care planning intervention in one study [41], and an end-of-life conversation game in one study [42]. Most programs were based on discussions, consultations, and interviews, just like in the RCTs [35,39-41], and some programs additionally provided education $[34,36]$. One of the programs used the same feedback form as that in the corresponding RCT [37], while another used the pathway tool [38] to catego-

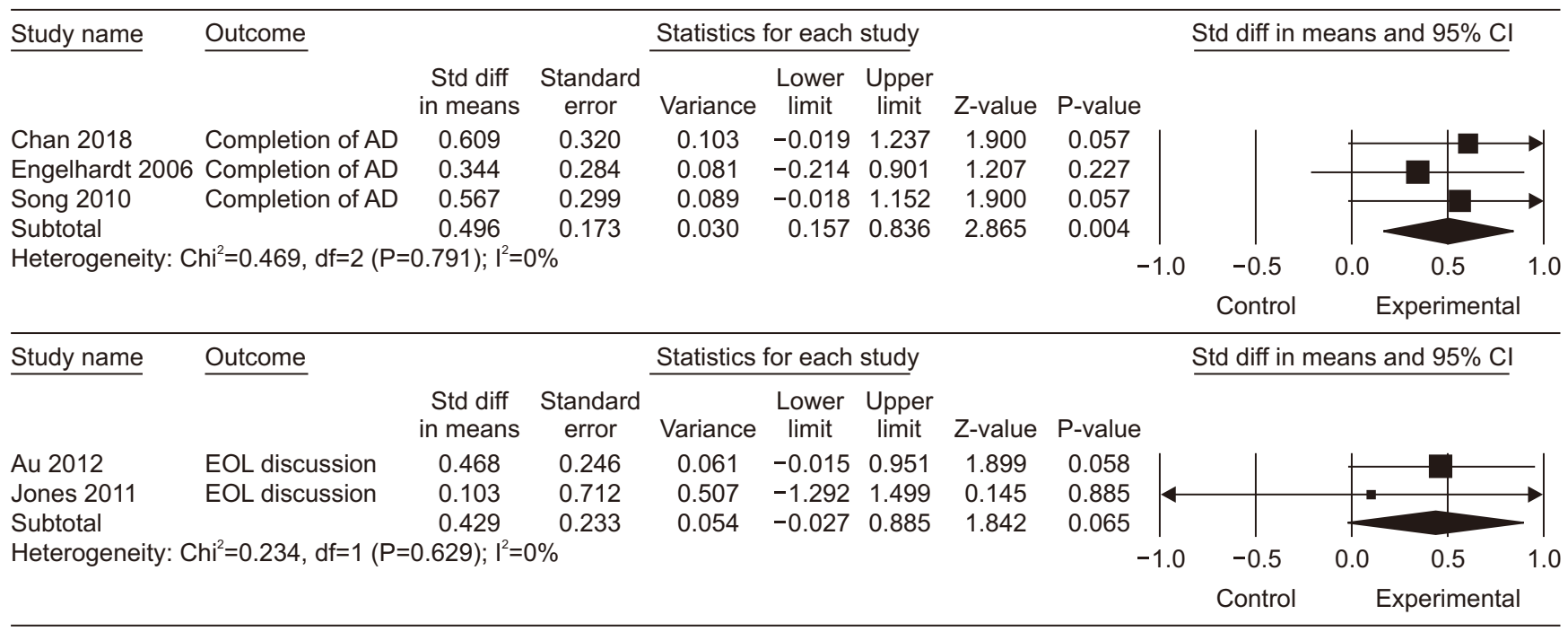

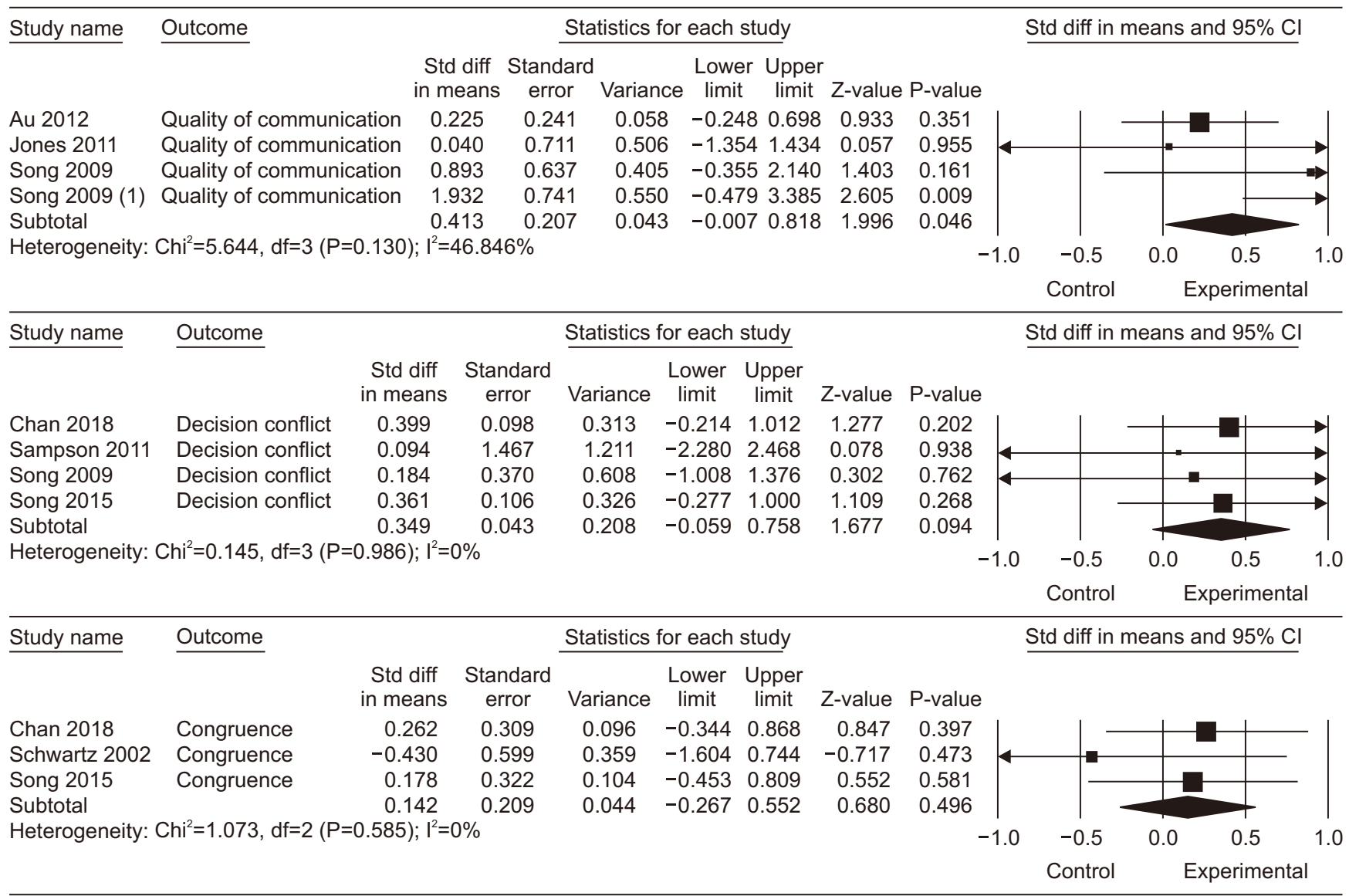

Figure 2. Forest plots of the included studies.

AD: advance directives, EOL: end-of-life. 
rize patients and help them designate a legal representative or document discussions on their wishes and to provide advice on the role of a patient's legal representative. Another intervention program utilized games [42], so that patients and their legal representatives could access the content easily. All the interventions included creating advance directives and documenting patients' wishes in the last step. Most interventions comprised a single session lasting from 20 minutes to 1.5 hours.

The outcome variables of the studies meeting the selection criteria of this study were the completion of advance directives and advance care plans, discussion of end-of-life care, quality of communication, decisional conflict, the patient's legal representative's confidence in decision-making, congruence regarding preferred care between the patient and the patient' $s$ legal representative, and congruence between preferences for care and delivered care.

\section{Meta-analysis outcomes of RCT studies}

The effect size of variables related to advance care planning interventions can be found in Figure 2. The three studies that analyzed documentation of advance directives and advance care plans as the primary outcome variables had no heterogeneity $\left(\mathrm{I}^{2}=0 ; \mathrm{P}=0.791\right)$, with an effect size of $0.496(95 \%$ CI: $0.157 \sim 0.836 ; Z=2.865 ; P=0.004)$. The two studies that analyzed end-of-life care discussions showed no heterogeneity $\left(\mathrm{I}^{2}=0 ; \mathrm{P}=0.629\right)$, with an effect size of 0.429 (95\% CI: $-0.027 \sim 0.885 ; \mathrm{Z}=1.842 ; \mathrm{P}=0.065)$. The four studies analyzing the quality of communication demonstrated a medium level of heterogeneity $\left(\mathrm{I}^{2}=46.859 ; \mathrm{P}=0.130\right)$, with an effect size of 0.413 (95\% CI: 0.008 0.818; $\mathrm{Z}=1.996 ; \mathrm{P}=0.046$ ).

In an analysis of the effect size of the secondary outcome variables by intervention, the four studies that analyzed conflicts in decision-making had no heterogeneity $\left(\mathrm{I}^{2}=0, \mathrm{P}=0.986\right)$, with an effect size of 0.349 (95\% CI: $-0.059 \sim 0.758 ; Z=1.677$; $\mathrm{P}=0.094)$. The three studies analyzing congruence regarding preferred treatment between the patient and patient's legal representative showed no heterogeneity $\left(\mathrm{I}^{2}=0 ; \mathrm{P}=0.585\right)$, with an effect size of 0.142 (95\% CI: $-0.267 \sim 0.552 ; Z=0.680$; $\mathrm{P}=0.496)$. One of the studies that analyzed the patient's legal representative's confidence in decision-making as a secondary variable was excluded from the meta-analysis because its data could not be entered into the CMA statistical analysis software. Since all measured variables had low heterogeneity, with $\mathrm{P}>0.10$, a fixed-effects model was applied for effect sizes between 0.142 and 0.496 .

\section{Publication bias outcomes}

Funnel plot and trim-and-fill analyses were performed to evaluate publication bias. The funnel plot analysis demonstrated a symmetrical funnel shape (Figure 3 ). The trimand-fill analysis results confirmed that no studies were added to correct the asymmetry and the effect size did not change compared to the correction. It was concluded that the studies analyzed herein were not affected by publication bias.

\section{DISCUSSION}

This study analyzed effect sizes through a systematic review and meta-analysis of previous experimental research to understand how intervention programs developed to assist in decision-making for end-of-life care affected advance care planning. Eighteen studies were finally selected for the analysis after reviewing a total of 4,668 studies found in the initial search.

The meta-analysis showed that advance care planning interventions had significant effects on the completion of advance directives and advance care planning, as well as communication quality, but no significant effect on discussions on endof-life care, decisional conflict, or congruence in preferred care between patients and their legal representatives. The outcome

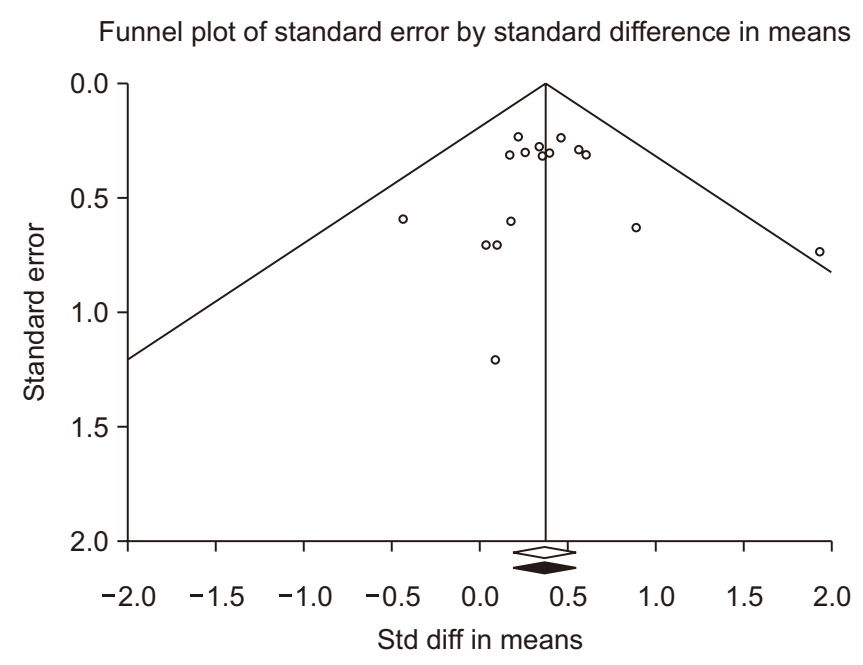

Figure 3. Funnel plot of the included studies. 
variable with the largest effect size was completion of advance directives and advance care plans. Many of the interventions for advance care planning involved conversations and discussions with patients or patient's legal representatives to understand the status of the patient's condition or their concerns and preferences in end-of-life care, with the creation of documents such as advance directives as the last step [26,28,32]. Including documentation of advance directives as part of the intervention seems to have yielded effective outcomes in the documentation of advance directives. A systematic review of the effects of advance care planning [43] also suggested that advance care planning can be effective in encouraging patients to prepare advance directives. Korea has seen a gradual increase in the proportion of patients who write advance directives since the Act on Decisions on Life-Sustaining Treatment came into force [10]. Applying interventions for advance care planning could encourage more people to prepare advance directives and facilitate shared decision-making among patients, family members, and medical staff.

Although the interventions had no significant effects on discussions on end-of-life care, decisional conflict, and congruence in preferred care between patients and their legal representatives, the overall heterogeneity was low. As a variable, measurements of discussions on end-of-life care reflect the extent to which discussions on end-of-life care took place from the patient's point of view. The study by Au et al. [25] used a patient-specific feedback form to confirm the requests of the patient and family for care and to receive feedback from the medical staff without face-to-face discussions. This kind of intervention may have demonstrated a non-significant impact due to a lack of face-to-face interventions with the patient, which would have prompted sufficient discussion. In the study by Song et al. [32] that probed the effects of the SPIRIT intervention on decisional conflict showed no difference in conflicts by time or group, while another study by Song et al. [33] proved that decisional conflict decreased over time. The SPIRIT intervention targeted both patients and their legal representatives, but focused on improving the decision-making ability of the patient's legal representative rather than that of the patient, which may explain why it did not have a significant effect on decisional conflict.

Advance care planning interventions had a small impact on the level of congruence between patients and patient's legal representatives on preferred treatment, with an effect size of 0.142 , which means that it was not statistically meaningful. When looking at the effects of this intervention in individual studies that measured this variable, Chan et al. [26] and Schwartz et al. [30] showed that the intervention was effective by demonstrating that patients and their legal representatives had higher congruence after the intervention, while Song et al. [33] showed partial improvement in congruence; however, the level was inconsistent across different times of measurement. Although the impact of advance care planning interventions was small in terms of aligning the opinions between patients and their legal representatives, the interventions were of clinical significance because they had positive effects on fostering agreement between patients and their legal representatives.

The interventions used in the studies that had a normal or high SMD (between 0.609 and 1.932) were the "Let me talk" program [26], the end-of-life planning intervention [31], and the SPIRIT intervention program [32]. These intervention programs commonly included the process of discussions and conversations about experiences of illness, life experiences, interests, and wishes regarding end-of-life care. The "Let me talk" program had meaningful effects on congruence between patients and their legal representatives on preferred care, decisional conflict, and documentation of advance directives and advance care plans. The end-of-life planning intervention was effective in promoting the documentation of advance directives. The SPIRIT intervention program demonstrated effectiveness for boosting satisfaction with communication and confidence in decision-making. These results imply that when writing advance directives, it is important for patients and their families to share opinions through conversations and to plan optimal end-of-life care together, taking the wishes and priorities of both parties into account.

The intervention programs used in the selected studies varied from single interventions involving discussions to comprehensive interventions including discussions and education. These interventions suggest ways to systematically approach delivering bad news and exchanging opinions on end-of-life care. These advance care planning interventions could be applicable in the Korean setting, where talking about death is a cultural taboo and decision-making is led by family members. In the 
family-centered decision-making process, the patient is often alienated from the decision-making or his or her opinions are disregarded if different from the opinion of the family. Interventions to promote advance care planning are expected to encourage communication about opinions among the patient, family members, and the medical staff, supporting them to explore each other's thoughts and reach a consensus. Such interventions are also likely to serve as guidelines for medical staff on how to start the dreaded conversation about death or lifesustaining treatment with patients.

This study assessed the methodological quality of the studies using the JBI appraisal tools [20], which deal with potential bias broadly, ranging from study design to implementation and analysis. The tools contain similar items to version 2 of the risk of bias tool by the Cochrane Group. They are also efficient for saving time on the literature review, in the same way that the RCT quality assessment checklist is helpful for conducting a systematic literature review [44].

The principal limitation of this study is that the intervention methods used in selected studies varied widely. Although the interventions all fall into the general category of advance care planning interventions, a detailed breakdown of the types of advance care planning interventions would be necessary given their widely different content and structures.

\section{CONFLICT OF INTEREST}

No potential conflict of interest relevant to this article was reported.

\section{SUPPLEMENTARY MATERIALS}

Supplementary materials can be found via https://doi. org/10.14475/kjhpc.2020.23.2.71.

\section{REFERENCES}

1. Shin DW, Lee JE, Cho B, Yoo SH, Kim S, Yoo JH. End-of-life communication in Korean older adults: with focus on advance care planning and advance directives. Geriatr Gerontol Int 2016;16:407-15.

2. Sun DS, Chun YJ, Lee JH, Gil SH, Shim BY, Lee OK, et al. Recognition of advance directives by advanced cancer patients and medical doctors in hospice care ward. Korean J Hosp Palliat Care 2009;12:20-6.

3. Kim HS, Shin SR. The influence of social support among community dwelling elderly and their attitude towards the withdrawal of life-sustaining treatment: a mediating effect of self-esteem. Korean J Adult Nurs 2017;29:373-81.

4. Kim MH, Chun JR, Hong SA. Factors of family function and self-efficasy influencing old patient's decision of advance directives attitude. J Convergence Information Technology 2016;6:123-9.

5. Ryu GH. Consideration on the life-sustaining treatment decision system. Legal Theory Practice Review 2019;7:191-211.

6. Kim SH. Factors influencing preferences of Korean people toward advance directives. Nurs Ethics 2011;18:505-13.

7. Lee SR, Shin DS, Choi YJ. Perceptions of caregivers and medical staff toward DNR and AD. Korean J Hosp Palliat Care 2014;17:66-74.

8. Kang NY, Park JY. Clinical characteristics of oncologic patients with DNR decision at a tertiary hospital. Korean J Hosp Palliat Care 2016;19:26-33.

9. Kim MH. The problems and the improvement plan of the hospice/palliative care and dying patient's decisions on life-sustaining treatment act. Korean J Hosp Palliat Care 2018;21:1-8.

10. Current Status of Life-Sustaining Treatment System [Internet] Seoul: National Agency for Management of Life-Sustaining Treatment; 2019 [cited 2019 Nov 7]. Available from: https://www.Ist.go.kr.

11. Koh YS. Current status of end-of-life care in Korean hospitals. J Korean Med Assoc 2012;55:1171-7.

12. Lee SB. Issues and tasks of the sog. Well-Dying Act. Kookmin Law Review 2017;29:311-40.

13. Kwon YO, Ahn SH. The attitude and perception on withdrawal of futile life sustaining treatment and patient self determination right among home care nurses. J Korean Bioethics Assoc 2013;14:53-66.

14. Kwon KE. A study on the model of life-sustaining treatment decision making through shared decision making at the end of life. J Korean Bioethics Assoc 2019;20:45-61.

15. Molloy DW, Guyatt GH, Russo R, O’Brien B, Bedard M, et al. Systematic implementation of an advance directive program in nursing homes: a randomized controlled trial. JAMA 2000;283:1437-44.

16. Chan HY, Pang SM. Let me talk-an advance care planning programme for frail nursing home residents. J Clin Nurs 2010;19:3073-84.

17. MacKenzie MA, Smith-Howell E, Bomba PA, Meghani SH. Respecting choices and related models of advance care planning: a systematic review of published evidence. Am J Hosp Palliat Care 2018;35:897-907. 
18. Rocque GB, Dionne-Odom JN, Huang C-HS, Niranjan SJ, Williams CP, Jackson BE, et al. Implementation and impact of patient lay navigator-led advance care planning conversations. J Pain Symptom Manage 2017;53:682-92.

19. Hickman SE, Nelson CA, Perrin NA, Moss AH, Hammes BJ, Tolle SW. A comparison of methods to communicate treatment preferences in nursing facilities: traditional practices versus the physician orders for life-sustaining treatment program. J Am Geriatr Soc 2010;58:1241-8.

20. Joanna Briggs Institute. Critical appraisal tools [Internet]. Adelaide: Joanna Briggs Institute; 2017 [cited 2019 April 30] . Available from: https://joannabriggs.org/ebp/critical_appraisal_tools.html.

21. Cohen J. Statistical power analysis for the behavioral science. 2nd ed. Hillsdale, NJ:Lawrence Erlbaum Associates;1988.

22. Borenstein M, Hedges LV, Higgins JPT, Rothstein HR. Introduction to meta-analysis. Chichester, West Sussex, U.K.; Hoboken:John Wiley \& Sons;2009.

23. Higgins J, Thomas J. Cochrane handbook for systematic reviews of interventions [Internet]. Version 6.0. Chichester (UK): John Wiley \& Sons, Ltd.; 2019 [updated 2019 July]. Available from: http://www.training.cochrane.org/handbook.pdf.

24. Duval S, Tweedie R. Trim and fill: a simple funnel-plot-based method of testing and adjusting for publication bias in meta-analysis. Biometrics 2000;56:455-63.

25. Au DH, Udris EM, Engelberg RA, Diehr PH, Bryson CL, Reinke LF, et al. A randomized trial to improve communication about end-of-life care among patients with COPD. Chest 2012;141:726-35.

26. Chan HY, Ng JS, Chan KS, Ko PS, Leung DY, Chan CW, et al. Effects of a nurse-led post-discharge advance care planning programme for community-dwelling patients nearing the end of life and their family members: a randomised controlled trial. Int J Nurs Stud 2018;87:26-33.

27. Engelhardt JB, McClive-Reed KP, Toseland RW, Smith TL, Larson DG, Tobin DR. Effects of a program for coordinated care of advanced illness on patients, surrogates, and healthcare costs: a randomized trial. Am J Manag Care 2006;12:93-100.

28. Jones L, Harrington J, Barlow CA, Tookman A, Drake R, Barnes K, et al. Advance care planning in advanced cancer: can it be achieved? An exploratory randomized patient preference trial of a care planning discussion. Palliat Support Care 2011;9:3-13.

29. Sampson EL, Jones L, Thune-Boyle IC, Kukkastenvehmas R, King M, Leurent B, et al. Palliative assessment and advance care planning in severe dementia: an exploratory randomized controlled trial of a complex intervention. Palliat Med 2011;25:197-209.

30. Schwartz CE, Wheeler HB, Hammes B, Basque N, Edmunds J, Reed G, et al. Early intervention in planning end-of-life care with ambulatory geriatric patients: results of a pilot trial. Arch Intern Med 2002;162:1611-8.

31. Song J, Ratner ER, Wall MM, Bartels DM, Ulvestad N, Petroskas D, et al. Effect of an end-of-life planning intervention on the completion of advance directives in homeless persons: a randomized trial. Ann Intern Med 2010;153:76-84.

32. Song MK, Ward SE, Happ MB, Piraino B, Donovan HS, Shields AM, et al. Randomized controlled trial of SPIRIT: an effective approach to preparing African-American dialysis patients and families for end of life. Res Nurs Health 2009;32:260-73.

33. Song MK, Ward SE, Fine JP, Hanson LC, Lin FC, Hladik GA, et al. Advance care planning and end-of-life decision making in dialysis: a randomized controlled trial targeting patients and their surrogates. Am J Kidney Dis 2015;66:813-22.

34. Ko E, Hohman M, Lee J, Ngo AN, Woodruff SI. Feasibility and acceptability of a brief motivational stage-tailored intervention to advance care planning: a pilot study. Am J Hosp Palliat Care 2016;33:834-42.

35. Morrison RS, Chichin E, Carter J, Burack O, Lantz M, Meier DE. The effect of a social work intervention to enhance advance care planning documentation in the nursing home. J Am Geriatr Soc 2005;53:290-4.

36. Payne KL, Prentice-Dunn S, Allen RS. A comparison of two interventions to increase completion of advance directives. Clin Gerontol 2009;33:49-61.

37. Reinke LF, Feemster LC, McDowell J, Gunnink E, Tartaglione EV, Udris E, et al. The long term impact of an end-of-life communication intervention among veterans with COPD. Heart Lung 2017;46:30-4.

38. Schamp R, Tenkku L. Managed death in a PACE: pathways in present and advance directives. J Am Med Dir Assoc 2006;7:339-44.

39. Schellinger S, Sidebottom A, Briggs L. Disease specific advance care planning for heart failure patients: implementation in a large health system. J Palliat Med 2011;14:1224-30.

40. Sinclair C, Auret KA, Evans SF, Williamson F, Dormer S, Wilkinson A, et al. Advance care planning uptake among patients with severe lung disease: a randomised patient preference trial of a nurse-led, facilitated advance care planning intervention. BMJ Open 2017;7:e013415.

41. Song MK, Donovan HS, Piraino BM, Choi J, Bernardini J, Verosky D, etal. Effects of an intervention to improve communication about end-oflife care among African Americans with chronic kidney disease. Appl Nurs Res 2010;23:65-72.

42. Van Scoy LJ, Green MJ, Reading JM, Scott AM, Chuang C, Levi BH. Can playing an end-of-life conversation game motivate people to engage in advance care planning? Am J Hosp Palliat Care 2017;34:754-61.

43. Houben CHM, Spruit MA, Groenen MTJ, Wouters EFM, Janssen DJA. Efficacy of advance care planning: a systematic review and metaanalysis. J Am Med Dir Assoc 2014;15:477-89.

44. Klugar M. A protocol is essential for a systematic review as randomization is for randomized controlled trials. JBI Database System Rev Implement Rep 2016;14:1-2. 